Document downloaded from:

http://hdl.handle.net/10251/48658

This paper must be cited as:

Canto, B.; Coll, C.; Sanchez, E.; et ál..(2014). On identifiability for chemical systems from measurable variables. Journal of Mathematical Chemistry. 52(4):1023-1035. doi:10.1007/s10910-013-0149-4.

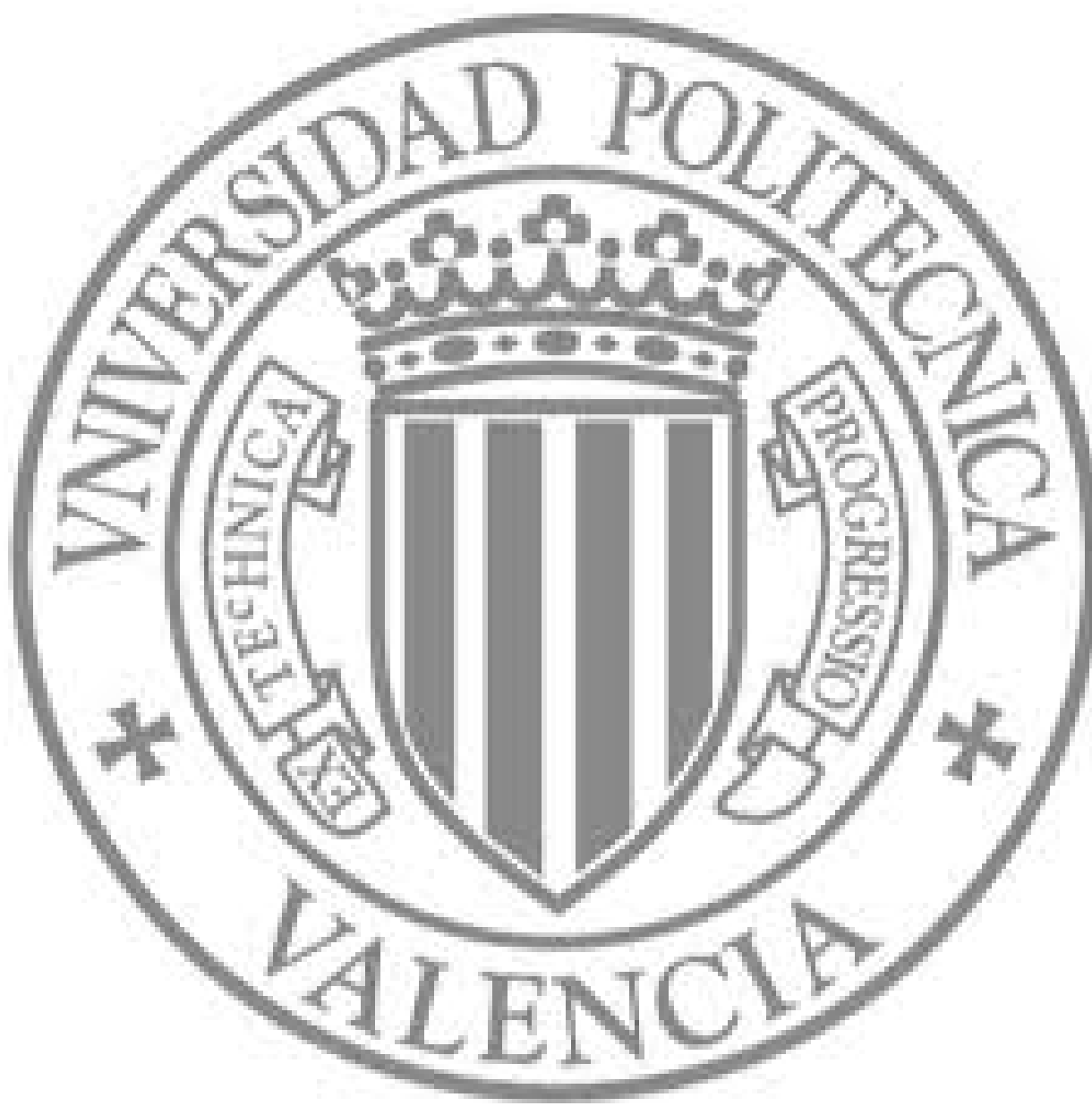

The final publication is available at

http://dx.doi.org/10.1007/s10910-013-0149-4

Copyright Springer Verlag (Germany) 


\title{
On identifiability for chemical systems from measurable variables
}

\author{
B.Cantó - S.C. Cardona - C.Coll • J. \\ Navarro-Laboulais • E. Sánchez
}

the date of receipt and acceptance should be inserted later

\begin{abstract}
The dynamics of the composition of chemical species in reacting systems can be characterized by a set of autonomous differential equations derived from mass conservation principles and some elementary hypothesis related to chemical reactivity. These sets of ordinary differential equations (ODEs) are basically non-linear, their complexity grows as much increases the number of substances present in the reacting media and can be characterized by a set of phenomenological constants (kinetic rate constants) which contains all the relevant information about the physical system. The determination of these kinetic constants is critical for the design or control of chemical systems from a technological point of view but the non-linear nature of the ODEs implies that there are hidden correlations between the parameters which maybe can be revealed with a identifiability analysis.
\end{abstract}

Keywords Continuous system · Structural properties · Identifiability • Observability

Mathematics Subject Classification (2000) $34 \cdot 93$

\section{B.Cantó}

Institut de Matemàtica Multidisciplinar, Universitat Politècnica de València, Escola Politècnica Superior d'Alcoi, Plaza Ferrándiz y Carbonell n.2, 03801, Alcoi, España

E-mail: bcanto@mat.upv.es

S.C. Cardona

Departament d'Enginyeria Química i Nuclear, Universitat Politècnica de València, Escola Politècnica Superior d'Alcoi, Plaza Ferrándiz y Carbonell n.2, 03801, Alcoi, España

C.Coll · E. Sánchez

Institut de Matemàtica Multidisciplinar, Universitat Politècnica de València, Camino de Vera s/n, 46022, Valencia, España

J. Navarro-Laboulais

Departament d'Enginyeria Química i Nuclear, Universitat Politècnica de València, Camino de Vera s/n, 46022, Valencia, España 


\section{Introduction}

The application of a chemical system in a technological domain implies the determination of the kinetic rate constants defined in the chemical reaction mechanism. A correct experimental design can save hours of laboratory work in the determination of these constants, and thus, the availability of mathematical analysis tools which allow the experimental design can be useful for the chemist community. In this way, the identifiability of the system plays an important role. This problem consists of the determination of all parameter sets which give the same input-output structure.

In Chemistry, the dynamics of the composition of chemical species in reacting systems can be characterized by a set of autonomous differential equations derived from mass conservation principles and some elementary hypothesis related to chemical reactivity. These sets of ordinary differential equations (ODEs) are basically non-linear, their complexity grows as much increases the number of substances present in the reacting media and can be characterized by a set of phenomenological constants (kinetic rate constants) which contains all the relevant information about the physical system. The determination of these kinetic constants is critical for the design or control of chemical systems from a technological point of view but the non-linear nature of the ODEs implies that there are hidden correlations between the parameters which maybe can be revealed with a structural identifiability analysis. The chemical irreversible reactions can be expressed as a particular class of the more general chemical reversible reactions. Although the former are more common in chemical systems, the reversible ones have the advantage that can be approached, under some experimental circumstances, to linear systems. Then in this work we propose to analyze a reversible chemical reacting network, assuming that initially it remains stationary in an equilibrium state. Then, we will imagine an experiment where this system is perturbed and that it will return to its same initial state.

The dynamics of chemical reaction are usually described by set of non linear first order differential equations. All the relevant information of the chemical mechanism is given by the stoichiometric matrix which contains the stoichiometric coefficients of all the chemical substances of all the elementary chemical reactions, allowing to deduce the production rate, $R_{j}$, for each species $[13,14]$. These differential equations are in the form of

$$
R_{j}=\frac{d z_{j}}{d t}=\sum_{i} v_{i j} k_{i} \prod_{j} z_{j}^{\left|v_{i j}\right|}
$$

where $v_{i j}$ are the stoichiometric coefficients, $k_{i}$ the kinetic rate constant of the $i$-th chemical reaction and $z_{j}$ the concentration of the $j$-th chemical substance.

The identifiability property of this model is studied in order to confirm the possible identifiable parameters of the model from a given set of experimental data. A procedure for the identification is proposed. This analysis identifies the parameters or the relationships between parameters which are accessible 
experimentally from an input-output pair. This analysis allows design experiments knowing a priori the relationships between the parameters. Given an experiment, it is possible to know the maximum achievable information of a model and it provides information to design optimization algorithms to obtain and to analyze the relative sensitivity of the parameters.

One of the purpose of this work is the structural identifiability analysis of autonomous systems such as equation (1) for chemical reactions. The aim is the use of this technique for an optimal experimental design. It is common in real chemical systems that for a given chemical reaction mechanism there are substances which cannot be measured, detected or simply are hypothetically predicted. If our objective is the accurate determination of all the kinetic rate constants, $k_{i}$, the unknown concentrations of (1) makes the system undefined in the sense that not all the state variables of the system are known. Then, our challenge is to derive the maximum information of the system from a partial knowledge of the state variables of the system.

In this work we consider a particular class of chemical reactions which can be linearized around an equilibrium point. Let us consider a global reversible chemical reaction which can be described by a reaction mechanism composed of $i$ elementary reversible reactions. Starting from an initial condition physically feasible, the system will attain a thermodynamic equilibrium point where the concentrations of all species are related with the equilibrium constants. If at this point the system is slightly perturbed, the equilibrium concentrations will change to another point and it will relax again to the original equilibrium point. Let consider that the chemical system can be described by

$$
\dot{z}=f(z, k) \quad z(0)=z_{0}
$$

where $f(\cdot)$ is a non-linear function of the concentrations $z$ 's. The equilibrium point is attained when the chemical potential of the system reach its minimum. At this point the composition is constant and thus

$$
0=f\left(z_{e}, k\right)
$$

Considering the first order series expansion of equation (2), the system can be approached to

$$
\dot{z} \approx f\left(z_{e}, k\right)+\sum_{j}\left(\frac{\partial f}{\partial z}\right)_{z_{e}}\left(z_{j}-z_{j e}\right) .
$$

Defining the deviation variable $x_{j}=z_{j}-z_{j e}$ and using the property (3), the system (2) is reduced to the linear system

$$
\dot{x}=A x
$$

where $A$ is the system matrix which depends on the kinetic rate constants and the equilibrium concentrations. The structural identifiability analysis will be applied to this linear system.

Under this framework, two chemical systems are analyzed in this paper. A first simple academic model where the methodology is shown and tested under 
different circumstances. The second example under analysis is based on the oxidation of bromide by ozone, a system with evident technological interest in drinking water treatment plants.

The organization of the paper is as follows. In Section 2, we introduce some definitions and state some preliminary results needed in later sections. In Section 3, we analyze a reversible chemical reacting network, assuming that initially it remains stationary in an equilibrium state. In Section 4, we apply the results of Section 3 to study an experimental chemical model and we give a discussion about the accuracy of results. Finally, in Section 5, some conclusions are given.

\section{Preliminaries}

Linear systems will play a key role in obtaining the results on identifiability, because we use chemical reactions whose mathematical model can be approximated to linear systems.

When we consider the impulse on one reactive the linear system is described by

$$
\dot{\mathbf{x}}=A(\mathbf{p}) \mathbf{x}+B u
$$

where $B=e_{i}$ being $e_{i}$ the canonical vector.

In this case, it is important to identify the parameters of matrix $A(\mathbf{p})$. Several authors have studied the identifiability problem using different techniques and some results on structural identifiability are given. In particular, if we consider the Markov parameters of system (4), $V_{j}(\mathbf{p})=A^{j}(\mathbf{p}) B, j \geq 0$, we can prove that the system is identifiable, that is all the parameters of the model can be known using experimental data (see [7]) and a characterization of structural identifiability of system (4) is given in [2].

Therefore, usually the parameters of the model are unknown and cannot be pre-specified, and need to be estimated from data collected experimentally by measuring the observable variables. In this step, first we must know the number of variables that we can hope to measure. The number of directly observable variables may influence in the identifiability of the system and even sometimes can miss the identifiability. Then it is important to obtain the minimum number of variables that must be measured to identify the chemical process. Now, this process can be described by this continuous linear control system

$$
\begin{aligned}
\dot{\mathbf{x}} & =A(\mathbf{p}) \mathbf{x}+B u \\
y & =C \mathbf{x} .
\end{aligned}
$$

In the above system the information on the observable or measured variables is obtained from the algebraic equations, that is, it is given by the structure of the matrix $C$. Then, the dynamic chemical system can be represented as a relationship between the observable o measurable output, $y$, and the perturbation, $u$. That is, we have an input-output model. The problem of the 
structural identifiability of the model consists of the determination of all parameter sets which give the same input-output structure. In this case, the Markov parameters that allows the study of the identifiability where we consider the measurable variables are defined by $V_{j}(\mathbf{p})=C A^{j}(\mathbf{p}) B, j \geq 0$.

The identification problem associated to variables that can be measured is related to determining the minimum number of rows of the matrix $C$ to assure the identifiability of all parameters of the system.

There exist several techniques to obtain the identifiability of the parameters. These techniques are useful depending on the model to analyze. In this study, the used technique is based in the study of Markov parameters of the system.

\section{Model AKL}

In this section, we propose to analyze a reversible chemical reacting network, assuming that initially it remains stationary in an equilibrium state. Let us consider this system given in figure 1 .

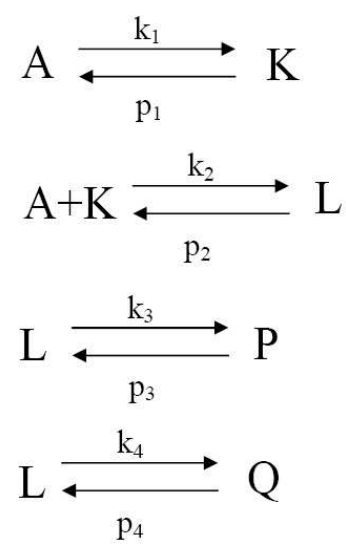

Fig. 1 Chemical reversible reacting system.

In this figure the direct and the reverse kinetic rate constants, $k_{i}$ and $p_{i}$ respectively, are indicated on each reaction. This example of reacting system has been set because includes several situations that can be encountered in typical chemical reaction mechanisms such as consecutive, competitive, first and second order chemical reactions. We expect that the identifiability analysis of such system will give some relevant information in more complex chemical systems about how the kinetic rate constants are related or the mathematical procedures that we will expect to need.

The dynamic model describing the internal structure of the reactions given in figure 1 is formulated theoretically using nonlinear state-space mathematical equations, depending on unknown parameters. That is, 


$$
\begin{aligned}
\dot{z}_{A} & =-k_{1} z_{A}+p_{1} z_{K}-k_{2} z_{A} z_{K}+p_{2} z_{L} \\
\dot{z}_{K} & =k_{1} z_{A}-p_{1} z_{K}-k_{2} z_{A} z_{K}+p_{2} z_{L} \\
\dot{z}_{L} & =k_{2} z_{A} z_{K}-\left(p_{2}+k_{3}+k_{4}\right) z_{L}+p_{3} z_{P}+p_{4} z_{Q} \\
\dot{z}_{P} & =k_{3} z_{L}-p_{3} z_{P} \\
\dot{z}_{Q} & =k_{4} z_{L}-p_{4} z_{Q}
\end{aligned}
$$

where $z_{A}, z_{K}, z_{L}, z_{P}$ and $z_{Q}$ are the concentrations of the reactive $A, K, L, P$ and $Q$ at time $t$, respectively.

The equilibrium point of the chemical reactions of this system is reached at the concentrations

$$
\begin{aligned}
& z_{1 e}=\frac{-\left(1+K_{1}\right)+\sqrt{\left(1+K_{1}\right)^{2}+8 K_{1} K_{2}\left(1+K_{3}+K_{4}\right) z_{10}}}{4 K_{1} K_{2}\left(1+K_{3}+K_{4}\right)} \\
& z_{2 e}=K_{1} z_{1 e} \\
& z_{3 e}=K_{1} K_{2} z_{1 e}^{2} \\
& z_{4 e}=K_{1} K_{2} K_{3} z_{1 e}^{2} \\
& z_{5 e}=K_{1} K_{2} K_{3} z_{1 e}^{2}
\end{aligned}
$$

where $z_{1_{0}}$ is the initial concentration of species 1 and the equilibrium constants are related to the kinetic rate constants through

$$
K_{i}=\frac{k_{i}}{p_{i}}, \quad i=1, \ldots, 4 .
$$

This system can be linearized around the equilibrium point of the system

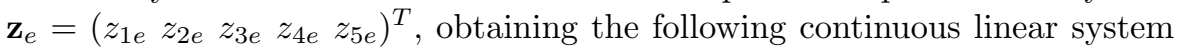
$\dot{\mathbf{x}}=A(\mathbf{p}) \mathbf{x}$ where the matrix $A(\mathbf{p})$ with $\mathbf{p}=\left(\begin{array}{lllllll}k_{1} & k_{2} & k_{3} & k_{4} & p_{1} & p_{2} & p_{3} \\ p_{4}\end{array}\right)$ is

$$
A(\mathbf{p})=\left(\begin{array}{ccccc}
-\left(k_{1}+k_{2} z_{2 e}\right) & p_{1}-k_{2} z_{1 e} & p_{2} & 0 & 0 \\
k_{1}-k_{2} z_{2 e} & -\left(p_{1}+k_{2} z_{1 e}\right) & p_{2} & 0 & 0 \\
k_{2} z_{2 e} & k_{2} z_{1 e} & -\left(p_{2}+k_{3}+k_{4}\right) & p_{3} & p_{4} \\
0 & 0 & k_{3} & -p_{3} & 0 \\
0 & 0 & k_{4} & 0 & p_{4}
\end{array}\right) .
$$

In particular, the above equations can model a reversible chemical reacting network in a batch reactor, see [6]. The equilibrium point can be perturbed by the injection, in impulse, of a given concentration of either component $A, K, L$, $P$ and $Q$. This injection is commonly employed as additional input variables. Moreover, the number of directly observable variables has influence in the identifiability of the parameters. Thus, it is necessary to know the measurable variables. In this case we consider the system

$$
\begin{aligned}
\dot{\mathbf{x}} & =A(\mathbf{p}) \mathbf{x}+B u \\
y & =C \mathbf{x} .
\end{aligned}
$$


where $A(\mathbf{p})$ is given by $(7)$ and $B=e_{i}, e_{i}$ is the canonical vector, $i=1, \ldots, n$ and $C$ is the matrix associated to the measurable variables. We are studying different cases according to several matrices $C$ and the results are given in the following theorem.

Theorem 1 Consider the system (8). If

(a) $B$ is equal to $e_{1}, e_{2}$ or $e_{3}$ and $C=\left(e_{2} e_{3} e_{4} e_{5}\right)^{T}$ or

(b) $B$ is equal to $e_{5}$ and $C=\left(\begin{array}{llll}e_{1} & e_{2} & e_{3} & e_{4}\end{array}\right)^{T}$,

then the system (8) is identifiable. Moreover, if

(c) $B=e_{4}$ and $C=\left(e_{3} e_{4} e_{5}\right)^{T}$ then the parameters $\left\{k_{2}, k_{3}, k_{4}, p_{2}, p_{3}, p_{4}\right\}$ are identifiable.

Proof. We consider two structured systems of type (8) with the vector parameters $\mathbf{p}$ and $\overline{\mathbf{p}}$, such that they have the same input-output behavior. It is known [2] that a system is identifiable if and only if, $i o(\mathbf{p})=i o(\overline{\mathbf{p}})$ implies that $\mathbf{p}=\overline{\mathbf{p}}$, where $i o(\cdot)$ denotes the input-output behavior of the system. That is, the system is identifiable if

$$
V_{j}(\mathbf{p})=V_{j}(\overline{\mathbf{p}}), j \geq 0 \Longrightarrow \mathbf{p}=\overline{\mathbf{p}} .
$$

Firstly, in each case we calculate the Markov parameters of the system (8), $V_{j}(\mathbf{p}), j=0 \ldots, 4$, since the size of the matrix $A(\mathbf{p})$ is $n=5$.

(a) We consider $B=e_{i}$ with $i=1,2,3$ and $C=\left(e_{2} e_{3} e_{4} e_{5}\right)^{T}$. We check that it is only necessary consider the equations $V_{j}(\mathbf{p})=V_{j}(\overline{\mathbf{p}}), j=0,1,2,3$ to assure the identifiability of the system.

(b) Now we consider $C=\left(\begin{array}{llll}e_{1} & e_{2} & e_{3} & e_{4}\end{array}\right)^{T}$. If $B=e_{5}$ we prove that the system is identifiable from the equations corresponding to $j=0,1,2,3$.

(c) Finally, if $B=e_{4}$ and $C=\left(e_{3} e_{4} e_{5}\right)^{T}$, using the Markov parameters corresponding to $j=0,1,2,3$, we identify the parameters $\left\{k_{2}, k_{3}, k_{4}, p_{2}, p_{3}, p_{4}\right\}$. Moreover we obtain a relationship between the parameters $k_{1}$ and $p_{1}$.

Note that if we perturb some components at the same time, the result is a linear combination of the results showed in the table, for each perturbed component individually. And an increase in the number of observable variables from those described in the table, does not improve the identifiability of the system.

Corollary 1 Consider the system (8). Then

1. If the concentration of the reactive $A K$ or $L$ is perturbed then to identify the system it is sufficient observe the reactives $K, L, P$ and $Q$.

2. If the concentration of the reactive $Q$ is perturbed then to identify the system it is sufficient observe the reactives $A, K, L$ and $P$.

3. If the concentration of the reactive $P$ is perturbed then to identify the maximum number of parameters it is sufficient observe $L, P$ and $Q$. In this case the identifiable parameters are $\left\{k_{2}, k_{3}, k_{4}, p_{2}, p_{3}, p_{4}\right\}$ and we obtain a relationship between $k_{1}$ and $p_{1}$. 
The table 1 shows the kinetic rate constants that can be identified from the Markov structural identifiability analysis, depending on the chemical species perturbed and the observable variables.

These results come from the application of the identifiability methodology after the linearization of the original nonlinear model. Consequently, it is pertinent to ask if the obtained results are useful from a practical point of view or if the results derived from the linear model are coherent with the structure of the nonlinear model.

The approach explored in this article was to fit the nonlinear model to the simulated data after the perturbation of some chemical species, and analyzing which kinetic rate constants are fitted, from a practical point of view, depending on the chosen observable variables. This last approach presents some restrictions that have to be taken into account and that limits the application of the conclusions beyond the system AKL. First, the results will be obtained in some local region of the space of parameters while any structural identifiability analysis is global. And second, the practical identifiability is affected by the sensitivity of the parameters in the model, which means that the number of parameters that are successfully fitted can be lower than those structurally identified. In any case, our purpose is to show if it is possible to fit more parameters of the nonlinear model than those identified from the approximated linear one.

The applied procedure can be described with the following steps:

1. Some values are assigned to the kinetic rate constants $k_{i}$ and $p_{i}$ and to the initial concentration $z_{1_{0}}$

$$
\begin{gathered}
k_{1}=4 s^{-1} ; k: 2=3 M^{-1} s^{-1} ; k_{3}=2 s^{-1} ; k_{4}=1 s^{-1} ; p_{1}=0.8 s^{-1} ; p_{2}=0.6 s^{-1} \\
p_{3}=0.4 s^{-1} ; p_{4}=0.2 s^{-1} ; z_{1_{0}}=1 M
\end{gathered}
$$

2. The equilibrium concentrations $z_{i e}$ are calculated from equations (6).

3. The initial conditions of the nonlinear ODE system (5) are fixed according to the chemical species perturbed in the following way

$$
z_{i_{0}}=z_{i_{e}}+M z_{i_{e}}
$$

where $M$ is a diagonal matrix which diagonal terms are the percentage of increase in the equilibrium concentration of each chemical specie perturbed.In this case a $10 \%$ has been applied.

4. The nonlinear ODE system (5) is solved using Matlab $2010 b$ and fixing a simulation time enough long for assuring that a new steady state is achieved (25s in this case).

5. Depending on the observable variables considered, only the above simulated concentrations corresponding to those observable variables are taken into account in the following optimization step.

6. The Levenberg-Marquardt optimization algorithm [8] has been used for fitting the nonlinear model (5) to the simulated concentrations of the observable chemical species. The success of the convergence of the least-squares 
minimization algorithm depends, among other factors, on the value of the initial parameters used in the iteration algorithm [9]. In this case, the initial parameters used are the $10 \%$ of the original ones defined in the step 1.

7. The fitted parameters are compared with those used in the simulation and only the fitted ones that differ less than a $5 \%$ from the simulated ones are considered as identified from a practical point of view.

The results can be seen in the table 1 , in comparison with the results obtained from the Markov structural identifiability analysis applied to the linearized model. In any analyzed case non identified parameters have been fitted, indicating that some of the information deduced from the linearized model can be translated to the nonlinear one. Conversely, some identified parameters have not been fitted, as could be expected taking into account the sensitivity of the parameters.

\begin{tabular}{|c|c|c|c|c|c|c|c|c|c|c|}
\hline $\begin{array}{c}\text { Perturbed } \\
\text { variables }\end{array}$ & Observed & \multicolumn{1}{|c|}{ Technique } & \multicolumn{3}{|c|}{ Identified parameters } \\
\hline$A$ & $A, K, L, P, Q$ & Identifiability & $\times$ & $\times$ & $\times$ & $\times$ & $\times$ & $\times$ & $\times$ & $\times$ \\
$A$ & $A, K, L, P, Q$ & Simulation & $\times$ & & & & $\times$ & & & \\
\hline$A$ & $K, L, P, Q$ & Identifiability & $\times$ & $\times$ & $\times$ & $\times$ & $\times$ & $\times$ & $\times$ & $\times$ \\
$A$ & $K, L, P, Q$ & Simulation & & & & & & & & \\
\hline$A$ & $L, P, Q$ & Identifiability & & $\times$ & $\times$ & $\times$ & & $\times$ & $\times$ & $\times$ \\
$A$ & $L, P, Q$ & Simulation & & & $\times$ & $\times$ & & & $\times$ & $\times$ \\
\hline \hline$K$ or $L$ & $A, K, L, P, Q$ & Identifiability & $\times$ & $\times$ & $\times$ & $\times$ & $\times$ & $\times$ & $\times$ & $\times$ \\
$K$ or $L$ & $A, K, L, P, Q$ & Simulation & $\times$ & $\times$ & $\times$ & $\times$ & $\times$ & $\times$ & $\times$ & $\times$ \\
\hline$K$ or $L$ & $K, L, P, Q$ & Identifiability & $\times$ & $\times$ & $\times$ & $\times$ & $\times$ & $\times$ & $\times$ & $\times$ \\
$K$ or $L$ & $K, L, P, Q$ & Simulation & $\times$ & $\times$ & $\times$ & $\times$ & $\times$ & $\times$ & $\times$ & $\times$ \\
\hline$K$ or $L$ & $L, P, Q$ & Identifiability & & $\times$ & $\times$ & $\times$ & & $\times$ & $\times$ & $\times$ \\
$K$ or $L$ & $L, P, Q$ & Simulation & & & $\times$ & $\times$ & & & $\times$ & $\times$ \\
\hline \hline$P$ & $A, K, L, P, Q$ & Identifiability & & $\times$ & $\times$ & $\times$ & & $\times$ & $\times$ & $\times$ \\
$P$ & $A, K, L, P, Q$ & Simulation & & $\times$ & $\times$ & $\times$ & & $\times$ & $\times$ & $\times$ \\
\hline$P$ & $K, L, P, Q$ & Identifiability & & $\times$ & $\times$ & $\times$ & & $\times$ & $\times$ & $\times$ \\
$P$ & $K, L, P, Q$ & Simulation & & $\times$ & $\times$ & $\times$ & & $\times$ & $\times$ & $\times$ \\
\hline$P$ & $L, P, Q$ & Identifiability & & $\times$ & $\times$ & $\times$ & & $\times$ & $\times$ & $\times$ \\
$P$ & $L, P, Q$ & Simulation & & & $\times$ & $\times$ & & & $\times$ & $\times$ \\
\hline \hline$Q$ & $A, K, L, P, Q$ & Identifiability & $\times$ & $\times$ & $\times$ & $\times$ & $\times$ & $\times$ & $\times$ & $\times$ \\
$Q$ & $A, K, L, P, Q$ & Simulation & & $\times$ & & $\times$ & & $\times$ & & $\times$ \\
\hline$Q$ & $K, L, P, Q$ & Identifiability & & $\times$ & $\times$ & $\times$ & & $\times$ & $\times$ & $\times$ \\
$Q$ & $K, L, P, Q$ & Simulation & & & & $\times$ & & & & $\times$ \\
\hline$Q$ & $L, P, Q$ & Identifiability & & $\times$ & $\times$ & $\times$ & & $\times$ & $\times$ & $\times$ \\
$Q$ & $L, P, Q$ & Simulation & & & & $\times$ & & & & $\times$ \\
\hline
\end{tabular}

Table 1 Theoretical and simulation results.

\section{Model Bromide/Bromate}

The second system under study is the chemical formation of bromate by an ozonation process in a drinking water treatment plant $[3,4]$. The ozonation is 
used in drinking water production as a disinfection step which can be complicated by the presence of bromide, $\mathrm{Br}^{-}$, reacting with the ozone, $\mathrm{O}_{3}$, potentially leading to the formation of bromates, $\mathrm{BrO}_{3}^{-}$, [10]. This substance is classified as possible carcinogenic and its production must be controlled below the $10 \mu \mathrm{g} / \mathrm{L}$ for drinking water following the recomendations of the WHO, EU and USEPA [16].

Our objective at this point is to analyze the real chemical mechanism under the perspective of the structural identifiability analysis to derive the information attainable from the model assuming the experimental restrictions. The chemical reaction mechanism of bromate formation is very complex and includes almost twenty elementary chemical reactions $[10,11,15,12]$. Here we will consider just the initial steps of the reaction between the ozone and the bromide. According [12] the reaction between the $\mathrm{Br}^{-}$and the $\mathrm{O}_{3}$ in acid media can be approached to

$$
\begin{gathered}
\mathrm{O}_{3}+\mathrm{Br}^{-} \underset{\mathrm{k}_{2}}{\stackrel{\mathrm{k}_{1}}{\rightleftarrows}} \mathrm{BrOOO}^{-} \\
\mathrm{BrOOO}^{-} \underset{\mathrm{k}_{4}}{\stackrel{\mathrm{k}_{3}}{\rightleftarrows}} \mathrm{HOBr}+\mathrm{O}_{2} \\
\mathrm{HOBr}+\mathrm{Br}^{-} \stackrel{\mathrm{k}_{5}}{\rightleftarrows} \mathrm{Br}_{2} \\
\mathrm{Br}_{2}+\mathrm{Br}^{-} \stackrel{\mathrm{k}_{7}}{\rightleftarrows} \mathrm{kr}_{3}^{-}
\end{gathered}
$$

Fig. 2 Bromide/bromate initial reaction mechanism.

For simplicity let us rewrite the chemical mechanism as

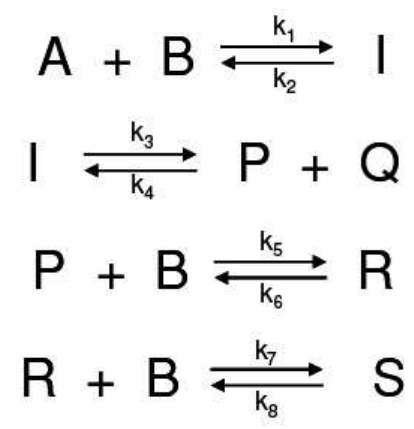

Fig. 3 Chemical reversible reacting system.

The equilibrium concentrations of this chemical system are given by the solution of the following system of algebraic equations derived from the mass 
action law

$$
\begin{aligned}
K_{1} z_{1 e} z_{2 e}-z_{3 e} & =0 \\
K_{3} z_{3 e}-z_{4 e} z_{5 e} & =0 \\
K_{5} z_{4 e} z_{2 e}-z_{6 e} & =0 \\
K_{7} z_{6 e} z_{2 e}-z_{7 e} & =0 \\
-2 z_{1_{0}}+z_{2_{0}} & =-2 z_{1 e}+z_{2 e}-z_{3 e}-z_{4 e}+z_{7 e} \\
3 z_{1_{0}}-z_{2_{0}} & =3 z_{1 e}-z_{2 e}+2 z_{3 e}+2 z_{4 e}+z_{6 e} \\
z_{1_{0}} & =z_{1 e}+z_{3 e}+z_{5 e}
\end{aligned}
$$

where $z_{1_{0}}$ and $z_{2_{0}}$ are the initial concentrations for the ozone and the bromide, respectively.

At this point a discussion about the physically known state variables and equilibrium constants must be done to define the structural identifiability problem. Not all the chemical species of the mechanism in the figure 2 can be measured or can be followed its deviation from the equilibrium point. Recall that the experiment modelled here considers that in a first stage the system evolves from the initial state to an equilibrium point. Then, the system is perturbed in a way that the concentrations of the chemical species change and the relaxation to the precedent equilibrium point is followed to derive the kinetic information of the system. The chemical species like $\mathrm{Br}^{-}$or $\mathrm{HOBr}$ cannot be followed easily in real time, but others like $\mathrm{O}_{3}, \mathrm{Br}_{2}$ or $\mathrm{Br}_{3}^{-}$can be followed with an spectrophometer $[1,5]$. Consequently, in an hypothetical experiment design only the state variables $x_{1}, x_{6}$ or $x_{7}$ or combinations of two of these state variables are accessible and the structural identifiability analysis is restricted to this knowledge. Additionally, neither all the equilibrium concentrations will be known again because the instrumental limitations for chemical species detection. For the system under study, the equilibrium concentration of the $\mathrm{O}_{3}$, the $\mathrm{O}_{2}$ and the $\mathrm{Br}_{2}$ or the $\mathrm{Br}_{3}^{-}$can be easily measured, i. e., $z_{1 e}$, $z_{5 e}$ and $z_{6 e}$ or $z_{7 e}$.

The dynamic model describing the internal structure of the reactions given in figure 3 is formulated theoretically using nonlinear state-space mathematical equations, depending on unknown parameters. That is,

$$
\begin{aligned}
& \dot{z}_{1}=-k_{1} z_{1} z_{2}+k_{2} z_{3} \\
& \dot{z}_{2}=-k_{1} z_{1} z_{2}+k_{2} z_{3}-k_{5} z_{2} z_{4}+k_{6} z_{6}-k_{7} z_{2} z_{6}+k_{8} z_{7} \\
& \dot{z}_{3}=k_{1} z_{1} z_{2}-k_{2} z_{3}-k_{3} z_{3}+k_{4} z_{4} z_{5} \\
& \dot{z}_{4}=k_{3} z_{3}-k_{4} z_{4} z_{5}-k_{5} z_{2} z_{4}+k_{6} z_{6} \\
& \dot{z}_{5}=k_{3} z_{3}-k_{4} z_{4} z_{5} \\
& \dot{z}_{6}=k_{5} z_{2} z_{4}-k_{6} z_{6}-k_{7} z_{2} z_{6}+k_{8} z_{7} \\
& \dot{z}_{7}=k_{7} z_{2} z_{6}-k_{8} z_{7}
\end{aligned}
$$


where $z_{i}$ are the concentrations of the components, that is $z_{1}=[A], z_{2}=[B]$, $z_{3}=[I], z_{4}=[P], z_{5}=[Q], z_{6}=[R]$ and $z_{7}=[S]$ at time $t$ and $k_{i}$ are the reaction constants.

This system can be linearized around the equilibrium point of the system $\mathbf{z}_{e}=\left(A_{e} B_{e} I_{e} P_{e} Q_{e} R_{e} S_{e}\right)^{T}$, where only are unknown $B_{e}$ and $P_{e}$. We obtain the following continuous linear system

$$
\begin{aligned}
\dot{\mathbf{x}} & =A(\mathbf{p}) \mathbf{x}+B u \\
y & =C \mathbf{x}
\end{aligned}
$$

where the matrix $A(\mathbf{p})$ is given by

$$
\left(\begin{array}{ccccccc}
-\alpha & -K_{1} & k_{2} & 0 & 0 & 0 & 0 \\
\alpha & -K_{1}-\phi-K_{7} & k_{2} & -\varphi & 0 & k_{6}-\gamma & k_{8} \\
\alpha & K_{1} & -k_{2}-k_{3} & K_{4} & \beta & 0 & 0 \\
0 & -\phi & k_{3} & -\varphi-K_{4} & -\beta & k_{6} & 0 \\
0 & 0 & k_{3} & -K_{4} & \beta & 0 & 0 \\
0 & \phi-K_{7} & 0 & \varphi & 0 & -k_{6}-\gamma & k_{8} \\
0 & K_{7} & 0 & 0 & 0 & \gamma & -k_{8}
\end{array}\right)
$$

where $K_{1}=k_{1} z_{1 e}, K_{4}=k_{4} z_{5 e}, K_{7}=k_{7} z_{6 e}, \alpha=k_{1} z_{2 e}, \beta=k_{4} z_{4 e}, \varphi=$ $k_{5} z_{2 e}, \phi=k_{5} z_{4 e}, \gamma=k_{7} z_{2 e}$ and the entries of vector parameter $\mathbf{p}$ are the reaction constants $k_{i}, i=1, \ldots, 8, B_{e}$ and $P_{e}$. And the state variables $\mathbf{x}$ represent the variable deviation of the concentration with respect to an equilibrium point.

In this case the perturbation variables are $x_{1}$ or $x_{2}$ and the observable variables can be $x_{1}, x_{6}, x_{7}, x_{1}$ and $x_{6}$ or $x_{1}$ and $x_{7}$. Moreover the equilibrium concentrations that we know are $z_{1 e}, z_{6 e}$ and $z_{5 e}$.

It is easy to check that we can identify more number of variables if we observe $x_{1}$ and $x_{7}$. In this case, we give the next result.

Theorem 2 Consider the system (9). If $B=\left(\begin{array}{ll}e_{1} & e_{2}\end{array}\right)$ and $C=\left(e_{1} e_{7}\right)^{T}$ then the parameters $\left\{k_{1}, k_{2}, k_{3}, k_{4}, k_{7}, k_{8}, z_{2 e}, k_{5} z_{4 e}\right\}$ are identifiable and a relationship between $k_{6}, k_{7}, k_{5} z_{4 e}$ and $k_{5} z_{2 e}$.

Corollary 2 Consider the system (9). If firstly the deviation variable of the concentration $A$ in the equilibrium point is perturbed and later the deviation variable of the concentration $B$ in the equilibrium point is perturbed then to identify the maximum number of parameters it is sufficient observe the deviation variables of the concentrations $A$, and $S$ in the equilibrium point. In this case the identifiable parameters are $\left\{k_{1}, k_{2}, k_{3}, k_{4}, k_{7}, k_{8}\right\}$, the concentration of the component $B$ in the equilibrium point and the product between $k_{5}$ and the concentration of the component $P$ in the equilibrium point. Moreover we obtain this relation $k_{6} k_{7}-k_{6}\left(k_{5} z_{4 e}\right)-\left(k_{5} z_{4 e}\right)\left(k_{5} z_{2 e}\right)$. 


\section{Conclusions}

In this paper we study two chemical systems, one of them is a reversible chemical reacting network, model AKL, and the another one represents the chemical formation of bromate by an ozonation process in a drinking water treatment plant. For that, we model and linearize the processes and we study their identifiability properties. In the AKL model we simulate the process in order to check if the results obtained on the identifiability matchs up with the simulation results.

In AKL model we obtain that it is not necessary to observe all the components to identify the parameters. Only if we perturb the component $\mathrm{P}$ we do not assure the identifiability of all parameters, but we can identify relationship between them. The simulations and parameter fitting procedure, in this case, has shown that no non identified parameter has been fitted which means that some information from the linearized model can be extrapolated to the nonlinear one.

In Bromide/Bromate model we only can perturb the variables $x_{1}$ or $x_{2}$ and the observable variables can be $x_{1}, x_{6}, x_{7}, x_{1}$ and $x_{6}$ or $x_{1}$ and $x_{7}$. The best result is given when we perturbed firstly $x_{1}$ and after $x_{2}$ and we observe $x_{1}$ and $x_{7}$. In this case we do not assure the identifiability of all parameters, but we can identify most of them and we give a relationship between the rest of them.

Acknowledgements This work has been partially supported by MTM2010-18228.

\section{References}

1. M.J. Almendral, A. Alonso And M.S. Fuentes, Development of new methodologies for on-line determination of the bromate, J. Environ. Monit. 11 (2009) 1381-1388.

2. A. Ben-Zvi, P.J. McLellan, K.B. MCAuley, Identifiability of linear time-invariant differential-algebraic systems. I. The generalized Markov parameter approach, Ind. Eng. Chem. Res. 42 (2003) 6607-6618.

3. T.P. BonACQUisti, A drinking water utility's perspective on bromide, bromate, and ozonation, Toxicology 221 (2006) 145-148.

4. R. Butler, A. Godley, L. Lytton and E. Cartmell, Bromate environmental contamination: Review of impact and possible treatment, Crit. Rev. Environ. Sci. Tech. 35 (2005) 193-217.

5. R. Butler, L. Lytton, A.R. Godley, I.E. Tothill and E. Cartmell, Bromate analysis in groundwater and wastewater samples, J. Environ. Monit. 7 (2005) 999-1006.

6. B. Cantó, S.C. Cardona, C. Coll, J. Navarro-Laboulais and E. Sánchez, Dynamic optimization of a gas-liquid reactor, J. Math. Chem. 50 (2012) 381-393.

7. B. CAntó, C. Coll AND E. SÁnchez, Identifiability of a class of discretized linear partial differential algebraic equations, Math. Problems Eng. (2011) 1-12.

8. A. Constantinides And N. Mostoufi, Numerical methods for chemical engineers with MATLAB applications, Alkis Constantinides and Navid Mostoufi, Upper Saddle River, Prentice Hall PTR, 1999.

9. P. Englezos And N. Kalogerakis, Applied parameter estimation for chemical engineers, New York, Marcel Dekker, 2001.

10. U. von Gunten, Ozonation of drinking water. Part II. Disinfection and by-product formation in presence of bromide, iodide or chlorine, Water Res. 37 (2003) 1469-1487. 
11. B. Legube, B. Parinet, K. Gelinet, F. Berne And J.-Ph. Croue, Modeling of bromate formation by ozonation of surface waters in drinking water treatment, Water Res. 38 (2004) 2185-2195.

12. Q. Liu, L.M. Schurter, C.E. Muller, S. Aloisio, J.S. Francisco and D.W. Margerum, Kinetics and Mechanisms of Aqueous Ozone Reactions with Bromide, Sulfite, Hydrogen Sulfite, Iodide, and Nitrite Ions, Inorg. Chem. 40 (2001) 4436-4442.

13. J.B. Rawling And J.G. Ekerdt, Chemical Reactor Analysis and Design Fundamentals, Nob Hill Pub., Madison, 2002.

14. W.E. Stewart and M. Caracotsios, Computer Aided Modelling of Reactive Systems, John Wiley and Sons, New York, 2008

15. P. Westerhoff, R. Song, G. Amy And R. Minear, Numerical kinetic models for bromide oxidation to bromine and bromate, Water Res. 32 (1998) 1687-1699.

16. World Health ORganization, Bromate in Drinking-water, Document WHO/SDE/WSH/05.08/78,

http : //www.who.int/water anitation $_{h}$ ealth/dwq/chemicals/en/(accesed26/07/12). 